\title{
An Extensive Review of Significant Researches on Medical Image Denoising Techniques
}

\author{
Mredhula.L \\ Research Scholar, \\ Sathyabama University, Chennai
}

\author{
M.A.Dorairangaswamy \\ Dean, Department of CSE, AVIT, \\ Paiyanoor Chennai
}

\begin{abstract}
In this day and age, digital images play a significant role in our day-to-day life. Digital images are utilized in a wide range of fields like medical, business and more. Besides, the digital images play a vital part in the medical field in which it has been utilized to analyze the anatomy. These medical images are used in the identification of different diseases. Regrettably, the medical images have noises due to its different sources in which it has been produced. Confiscating such noises from the medical images is extremely crucial because these noises may degrade the quality of the images and also baffle the identification of the disease. Hence, denoising of medical images is indispensable. Researchers have recognized this issue and have provided lots of paradigms and techniques for use in the medical image denoising process. In this paper, an extensive review on denoising of medical images is presented together with the classification of medical images into either Radiographic or Ultrasound or MRI or CT image. In addition, a brief description on the digital images and medical images is presented. A concise note on Radiography, Ultrasound, MRI and CT images is also presented.
\end{abstract}

\section{Keywords}

Digital images, medical images, Radiography, Ultrasound, Medical Resonance Image (MRI), Computed Tomography (CT) image, denoising

\section{INTRODUCTION}

Digital images play a significant part in day to-day applications, [13]. Although the manufacturing technology of the electronic components (such as digital cameras, camera phones, etc) has been improved, the captured images still endure from several distortions. Among many distortions noise is one of the most important, and can be introduced by several sources such as: the recording medium (film, digital sensor), transmission medium, and measurement and quantization errors [24]. The intensity of impulse noise has the tendency of being either relatively elevated or low down. So, it could damage the image quality severely and cause some loss of image information details [23]. Due to additive white Gaussian noise which can be caused by poor image acquisition or by transferring the image data in noisy communication channels, distortion is one of the most prevalent cases. Impulse and speckle noises are comprised in other types of noises [13]. The image obtains a mottled, grainy, textured or snowy appearance with the presence of noise. Therefore, in the case of recovering an original image from noisy image, an irresistible interest has been observed in recent years [14]. The challenge of removing the noise from images has a sturdy history [11]. As a corollary, in order to build quantitative post-processing more robust and efficient, image processing procedures often entail removing image artifacts in advance [17].

The field of image processing has seen much research and progress since 1964, when the pictures of the moon transmitted by Ranger 7 were processed by a computer to correct various types of image distortions [4]. Generally, for modification and analysis of an image, image processing refers to a broad class of algorithms. During acquisition, post-processing, or rendering/visualization, Image Processing refers to the initial image manipulation [25]. For converting the captured RGB image found from the real source, preprocessing steps are essential so that they can be capable for performing any binary operations onto it [26]. Image processing modifies pictures to develop them (enhancement, restoration), extract information (analysis, recognition), and change their structure (composition, image editing) [2]. Image processing is exploited for improving the visual appearance of images to a human viewer and preparing images for measurement of the features and structures present [3]. In image pre-processing Denoising, Restoration, Pre-Segmentation, Enhancement, Sharpening and Brightness Correction are some of the steps comprised [27].

Noise reduction is an important step for any complicated algorithms, in computer vision and image processing [11] Denoising is necessary and the initial step to be taken prior to the image data is analyzed. It is essential to apply an efficient denoising technique, to compensate such data corruption [13]. The effort of image denoising is to improve an image that is cleaner than its noisy observation. Therefore, a substantial technology in image analysis is noise reduction and the initial step to be taken prior to images is analyzed [30]. Image denoising removes additive noise and retains the important signal features as much as possible [29]. Denoising of images is classically done with the following process: The image is transformed into some domain where the noise component is recognized without difficulty, to eliminate the noise, a thresholding operation is then applied, and finally to reconstruct a noise-free image, the transformation is inverted [28].

Initially, image restoration, subsequently, the processing of data for autonomous machines perception and at last the processing of images for improvement in human perception for example comparison or feature extraction are the three primary application areas. In medicine, for supporting diagnosis and research image processing techniques have been exploited [4]. In areas like Computer Vision, Image processing operations like smoothing and edge detection, and many more are very widely utilized [1]. In the medical field, images used for the diagnosis process plays a significant role. Therefore, denoising which removes noise from images is an important step in medical image processing. In this paper, we present a comprehensive review of the very important researches available in the literature for medical image processing, along with their denoising and analysis methods. For diagnosing the reviewed researches are classified according to the type of medical imaging technology employed. The remaining part of the paper is organized as follows: Section 2 describes medical image processing in detail. Sections 2.1, 2.2, 2.3 and 2.4 present a concise description on Radiography, Ultrasonography, MRI and 
CT images respectively. A broad review of medical image denoising is presented in section 3. Directions for future research are briefly outlined in section 4 and section 5 concludes the paper.

\section{MEDICAL IMAGE PROCESSING}

The influence and effect of digital images on modern society is unbelievable, and image processing is now a critical component in science and technology. In analysis methods and computeraided diagnosis, the rapid progress in computerized medical image reconstruction, and the associated developments, has propelled medical imaging into one of the most important subfields in scientific imaging [31]. In Medical image processing, the area of interest is being raised. It comprises an extensive range of methods and techniques, initiating with the acquisition of images by exploiting specialized devices, image enhancement and analysis, to 3D model reconstruction from 2D images [10]. For performing segmentation and for extracting important information an image is captured, digitized and processed in medical imaging [6]. Usually, Medical images are of low contrast and due to various acquisitions they have a complex type of noise frequently, transmission storage and display devices are also because of application of diverse types of quantization, reconstruction and enhancement algorithms [16].

The image processing techniques make it feasible, to extract meaningful information from medical images [15]. The main objective of medical imaging is to acquire a high resolution image with as much details as possible for the sake of diagnosis [5]. To achieve the finest possible diagnoses it is necessary that medical images be sharp, clear, and free of noise and artifacts. One of the major challenges in the study of medical imaging is noise removal in these digital images. Subsequently they could mask and blur important but delicate features in the images, the noise in medical images creates a problem; many proposed denoising techniques have their own problems [12]. medical image processing and analyzing focuses on three major research fields such as Structural Imaging, Functional Imaging, and Molecular [8]. The medical images such as X-ray Radiography, Ultrasonography, Computerized Tomography (CT) and Magnetic Resonance Imaging (MRI) [9] are conversed below.

\subsection{Radiography}

Digital Radiography (DR) has been exploited to portray a digital $\mathrm{x}$-ray imaging system that reads the transmitted $\mathrm{x}$-ray signal immediately after exposure with the detector in a place. Every digital detectors generally produce an output signal, in the form of electrons, which signify a quantity of charge that matches to the number of $\mathrm{x}$-rays absorbed in a given detector element (del) in the detector. The charge is then converted to a digital value, for storage in the image matrix [20]. As regards testing data and the ease of the testing method especially the Xray radiographic testing method is superior. Though, using an $\mathrm{X}$-ray film, it is hard to recognize the presence of defects, defect type, defect shape, etc. when the defect is minute [32]. In an extensive variety of imaging tasks such as chest, musculoskeletal, genitourinary etc, Digital Radiography is exploited [33].

\section{Advantages}

$>$ No film or chemicals based on silver are required to treat film.

$>$ Decreased film storage expenses since images can be warehoused digitally.

$>$ Computed radiography frequently necessitates lesser retakes because of under- or over-exposure which leads to reduced complete dose to the patient.
$>$ Image procurement is remarkably faster - image previews can be attainable in below 15 seconds.

$>$ A extensive variety of thicknesses may be reviewed in one exposure by modifying image brightness and/or contrast, , unlike traditional radiography based on film, which may require another exposure or multiple film speeds in one exposure to comprise extensive thickness range in a component.

$>$ Images can be enhanced digitally to support in understanding.

$>$ Images can be warehoused on disk or consigned for off-site appraisal.

$>$ Ever developing technology makes the CR more inexpensive today than ever. With Chemicals, dark room warehousing and staff to arrange them, a CR could be purchased for the same monthly price at the same time being environmentally reasonable, based on the magnitude of the Radiographic Operation.

\subsection{Ultrasonography}

A recent technique Ultrasonography is applied to study typical and atypical structures of diverse body organs. To examine organs which cannot be observed physically by other methods, it is a valuable tool. In determining the size and presence of lesions in the body organs it is very helpful. Using Ultrasonography apparatus Ultrasound waves are transmitted into the body and detect reflected waves from the body. Since the body organs have diverse densities, they vary in their capability to reflect sound waves [34]. Harmonic component, which is produced by tissues or contrast agents, is exploited by Ultrasound imaging. On diagnosis Ultrasound made a reasonable impact specially cardiovascular, internal organs and peripheral circulation. The incapability to penetrate air, obstructing the diagnosis in abdomens with excessive gas or chest diseases is the major drawback of ultrasound [18].

\section{Advantages}

$>$ It pictures muscle, soft tissue, and bone surfaces very well and is particularly valuable for relating the interfaces between solid and fluid-filled spaces.

$>$ It renders "live" images, where the operator can dynamically choose the enormously valuable portion for analysis and documenting modifications, often permitting fast diagnoses. Additionally, live images permit for ultrasound-guided biopsies or injections, which can be impractical with other imaging modalities.

$>$ It reveals the structure of organs.

$>$ It has no known long-term side effects and barely ever produces any distress to the patient.

$>$ Equipment is widely available and relatively flexible.

$>$ Miniature, effortlessly carried scanners are existing; tests can be conducted at the bedside.

> Comparatively economical than other modes of analysis, like computed X-ray tomography, DEXA or magnetic resonance imaging.

$>$ Spatial resolution of high frequency ultrasound transducers is superior to majority of other imaging modalities.

$>$ Through the utilization of an Ultrasound research interface, an ultrasound device can provide a comparatively cost-efficient, real-time, and adjustable method for acquiring data necessary for special research uses for tissue characterization and invention of new image processing methods

\subsection{Medical Resonance Imaging (MRI)}

To produce detailed sectional images of the body in any 
imaging plane, Magnetic resonance imaging (MRI) is used. MRI does not employ ionizing radiation but exploits radiofrequency (RF) fields when compared to the $\mathrm{x}$-ray based medical diagnostic techniques. Therefore, than the ionizing radiation-based imaging modalities, the modality is considered to have less health effects [22]. When the patient is positioned in the magnetic field, his /her positively charged hydrogen atoms become almost uniformly aligned. To deflect the aligned protons, a radiofrequency is then pulsed into the body. When the protons return to their original position energy is released, the strength of which is proportional to the number of aligned hydrogen protons that were deflected. To form images of scanned area the computer processes the data [18].

\section{Advantages}

$>$ MRI scan is not injurious to the patient

$>$ MRI utilizes intense magnetic fields and non-ionizing radiation in the radio frequency range, unlike $\mathrm{CT}$ scans and conventional X-rays, which utilize ionizing radiation.

$>$ MRI contrast agents are excellently handled and are much less probable than $\mathrm{x}$-ray contrast agents to produce allergic reactions or change kidney function.

$>$ MRI acquirements can be programmed to encode for diverse physiological events such as speed of moving tissue or blood, diffusion of water (helpful in identifying stroke)

$>$ MRI causes sectional images of alike resolution in any projection without transporting the patient.

$>$ Remarkable explanation of anatomic structures is obtained from inbuilt high levels of contrast resolution.

$>$ The images produced by MRI are extremely exhaustive, comprehensive and accurate much more so than other cardiac imaging tests.

\subsection{Computed Tomography (CT) images}

Based on acquisition x-ray beam geometry, Computed Tomography can be alienated into 2 categories namely: fan beam and cone beam. An x-ray source and solid-state detector are mounted on a rotating gantry in fan-beam scanners. Data are acquired by transmitting a narrow fan-shaped $x$-ray beam through the patient [36]. Haemorrhage and other lesions that may replicate stroke such as tumors, subdural/extradural haematomas and abscesses can be detected by Computed Tomography (CT) [35]. Especially for low-risk patients with a long life expectancy, Dose Reduction is a significant topic. However, reduction of the radiation dose will increase the amount of photon noise in CT images, which will degrade the image quality. Though there are numerous other effects that influence CT image quality, such as background noise and subject movement, photon noise contributes the most to CT image quality degradation. A clear relation between an increase in noise and a reduction in diagnostic accuracy and vice versa, i.e., a lower amount of noise will improve the diagnostic quality has been studied through these results [21].

\section{Advantages}

$>$ Speed.

$>$ Relatively reduced radiation exposure to the patient.

$>$ Comparatively small scanning time.

$>$ The lungs can be pictured in less than a minute.

$>$ CT totally prevents the superimposition of images of structures beyond the region of interest.

$>$ Dissimilarities between tissues that vary in physical density by below $1 \%$ can be perceived due to the inbuilt high-contrast resolution of CT.
$>$ Data from a single CT imaging method containing either many adjoining or one helical scan can be perceived as images in the axial, coronal, or sagittal planes, based on the diagnostic action.

$>$ Improved soft tissue contrast and cross-sectional imaging potential.

> The CT scan method is noninvasive and pain free, and is normally fast and comfortable for majority of patients.

$>$ It is accurate because CT scan yields an extremely clear depiction of where a tumor or other problem is situated to a doctor and whether it has spread, it can assist the doctor in scheduling a biopsy, surgery, radiation or other therapy with better accuracy.

$>$ The CT scan is the most comprehensive, and can yield the most comprehensive depiction of what's taking place within a patient's body to a doctor. They are especially valuable and broadly utilized in diagnosing cancer.

\section{REVIEW OF SIGNIFICANT RESEARCHES ON MEDICAL IMAGES}

An extensive range of research methodologies is employed for the denoising process on medical image is presented here. The reviewed researches are classified based on the denoising process on different types of images such as Radiography, Ultrasound, MRI and CT images.

\subsection{Radiography}

Based on splitting input images into two or three frequency channels, current digital radiography systems mostly utilize unsharp masking like enhancement algorithms. A fine detail enhancement as well as processing of global contrast has been allowed by Martin Stahl et al. [37]. However, medium size structures were not accessible. In extension of a standard algorithm of such type, they have developed and test an enhancement algorithm on the basis of hierarchically repeated unsharp masking, resulting in a multi-scale architecture permitting consistent access to structures of all sizes. By a pyramid-architecture, their algorithm has decomposed the radiograph, isolating it into eight or more channels representing structures of dissimilar sizes, known as "scales". By suitable nonlinear processing, weakly contrasting structures are then enhanced at each scale. The superior performance and high acceptance of their processing has been strongly indicated by the outcomes.

Based on a multi-scale decomposition of the images, Contrast enhancement of radiographies has recently proven to be a far more versatile and efficient method than regular unsharpmasking techniques, while containing these as a subset. The performance of two multi-scale methods, namely the Laplacian Pyramid and the Fast Wavelet Transform (FWT) are compared by Sabine Dippel et al. [38]. They have noticed that the enhancement based on the FWT have suffered from one serious drawback, the introduction of visible artifacts when large structures were enhanced strongly. When compared, the Laplacian Pyramid has allowed a smooth enhancement of large structures, such that visible artifacts were avoided. Merely for the enhancement of very small details, for denoising applications or compression of images, over the Laplacian Pyramid, the FWT have some advantages.

A popular diagnostic imaging method is Digital radiography. In obtaining as much simply interpretable diagnostic information as possible with reasonable absorbed doses of ionizing radiation, Denoising and enhancement have a significant 
potential. The computational efficiency of the denoising and enhancement becomes significant due to the escalating usage of high resolution and high precision images with a limited number of human experts. For fulfilling the requirements of digital X-ray image enhancement, Hakan Oktem et al. [39] have proposed a local adaptive image enhancement and simultaneous denoising algorithm. Without distorting the image and within a reasonable execution time, the algorithm has augmented the visibility of relatively non significant details. The algorithm was based on the modification of the wavelet transform coefficients via a point wise nonlinear transformation and reconstructing the enhanced image from the modified wavelet transform coefficients. In software, the implementation of algorithm was simple, quick, and universal.

Giakos et al. [40] have presented the design of a high-efficiency optical polarimetric system for imaging of cracks and structural defects of a rotating metallic shaft. The experimental outcomes have obviously indicated that high signal-to-noise ratio signals were attained, in order that it minimized the exploitation of processing techniques.

Pierre Gravel et al. [41] have developed a method to study the statistical properties of the noise found in various medical images. The method was exclusively designed for types of noise with uncorrelated fluctuations. Generally in the physical processes of imaging rather than in the tissue textures, such signal fluctuations has been originated. To degrade medical images, various types of noise has been contributed; the overall noise was generally assumed to be additive with a zero-mean, constant-variance Gaussian distribution. However, depending on external parameters related to the image acquisition protocol, statistical analysis has suggested that the noise variance was better modeled by a nonlinear function of the image intensity. To extract the relationship between image intensity and the noise variance and to evaluate the corresponding parameters, they have presented a method. To magnetic resonance images with diverse acquisition sequences and to several types of X-ray images the method was applied effectively.

For optimizing X-ray radiographic imaging, Mark Winslow et al. [42] have developed a software package, incorporating two computational patient phantoms. To simulate the scattered portion of an X-ray system using the Electron Gamma Shower National Research Council (EGSnrc) Monte Carlo code, a tomographic phantom, visible photographic Man tomographic phantom (VIP-Man), constructed from Visible Human anatomical color images was utilized. Using the projection raytracing method through the Visible Human CT data set, the primary portion of an X-ray image was simulated. The software has been simulated quantum noise, blurring effects, lesions, detector absorption efficiency, and other imaging artifacts to produce a realistic image. For upcoming observer studies and image quality analysis, the primary and scattered portions of an $\mathrm{X}$-ray chest image were combined to form a final image. From the Monte Carlo simulations, absorbed doses in organs and tissues of the segmented VIP-Man phantom were also obtained. They have offered the methods of the simulator and preliminary consequences.

Triet Le et al. [43] have proposed a variational model to denoise an image corrupted by Poisson noise. Similar to the ROF model, total-variation regularization has been utilized by the model, which preserves edges. Unlike the ROF model, a data-fidelity term that was suitable for Poisson noise was employed by their model. The result was that precisely like Poisson noise, the strength of the regularization was signal dependent. While preserving low-contrast features in regions of low intensity, noise of varying scales was removed by their model.

Frosio et al. [44] have proposed a filter to restore radiographic images corrupted by impulsive noise. It was based on a switching scheme where all the pulses were detected initially and then corrected through a median filter. The pulse detector was based on the hypothesis that the major contribution to image noise was offered by the photon counting process, with some pixels corrupted by impulsive noise. By an adequate mixture model, such statistics was described. The filter was also able to reliably estimate the sensor gain. On both synthetic and real images, its operation has been verified; the superiority of the proposed approach has been demonstrated with the experimental results in comparison with more traditional methods.

The most effective method for the early detection of breast diseases is X-ray mammography. Since mammograms are of low-contrast and noisy, the typical diagnostic signs such as micro calcifications and masses are tricky to detect. To screen mammograms, Krishnamoorthy et al. [45] have proposed an algorithm for image denoising and enhancement in Orthogonal Polynomials Transformation (OPT) for radiologists. By a scale factor called OPT scale factor, they have scaled a set of OPT edge coefficients to another set. The second set of coefficients was then inverse transformed resulting in contrast enhanced image. Applications of the proposed method to mammograms with delicate lesions were exposed. They have compared the results to those obtained by the Histogram Equalization (HE) and the Unsharp Masking (UM) methods, to authorize the effectiveness of the proposed method. Their preliminary results have strongly suggested that over the HE and UM methods, the proposed method has presented significantly improved enhancement potential.

\subsection{Ultrasonography}

Su Cheol Kang et al. [46] have proposed a simple and effective filter design based on multi scale wavelet denoising method for image denoising and contrast enhancement. With small magnitude, the Wavelet threshold algorithms have been replaced the wavelet coefficients by zero and shrink the other coefficients. Since wavelet coefficients have characterized the local regularity of a function, it was basically a local procedure. Once estimating the distribution of noise within echocardiographic image, it was applied to fitness Wavelet threshold algorithm. Corresponding to the standard SNR in case of additive noise the estimation of the speckle noise level in coherent imaging has utilized the S/MSE ratio. Their experiments have exposed that the optimal threshold level were based on the spectral content of the image. To over-estimate the noise standard deviation estimation performed at the finest level of the DWT, High spectral content has been tended. As a result, to obtain the optimal S/MSE, a lower threshold parameter was required. The standard WCS theory have predicted threshold that has depended only on the number of signal samples.

Suiren Wan et al. [47] have proposed a robust method for deconvolution of high-frequency ultrasound skin images using a combination of higher-order spectral methods and wavelet analysis. In their effort, for the sake of ease, the axial and lateral deconvolution methods were decoupled. The finest resolution possible in the axial direction has depended on the sampling frequency, for a given tissue. In the case of transducer, the resolution improvement was even higher in the axial direction compared to those of the other two transducers, although the same sampling rate was used for all transducers. Since the axial and lateral signals were not entirely decoupled, it was most liked and each A-scan was not strictly a representation of one 


\section{line of data.}

A nonlinear edge enhanced anisotropic diffusion model has been proposed by Shujun Fu et al. [48] to lessen ultrasonic speckle noise while preserving the edges, local details and ultrasonic echoic bright strips. Their technique encompasses the advantages of denoising and conserving significant features and organ surfaces well, which has a large potential in ultrasonic imaging enhancement and in assisting automated segmentation/ calculation techniques.

Based on texture analysis, image quality evaluation metrics, and visual evaluation, Christos Loizou et al. [49] have proposed a comparative evaluation of despeckle filtering by medical experts in the assessment of 440 (220 asymptomatic and 220 symptomatic) ultrasound images of the carotid artery bifurcation. Based on local statistics, median filtering, pixel homogeneity, geometric filtering, homomorphic filtering, anisotropic diffusion, nonlinear coherence diffusion, and wavelet filtering, they have evaluated a total of 10 despeckle filters. The outcomes of their study suggested that the first order statistics filter lsmv, have offered the best performance, followed by the geometric filter gf $4 \mathrm{~d}$, and the homogeneous mask area filter lsminsc. On the basis of the statistics of the extracted texture features, the class separation between the asymptomatic and the symptomatic classes has been enhanced by those filters, only provided a marginal progression in the classification success rate, and the visual assessment carried out by the two experts has been enhanced. In addition, for despeckling asymptomatic images, filters 1 smv or gf4d was used in which the expert was generally concerned in the plaque composition and texture analysis; and filters $1 \mathrm{smv}$, gf $4 \mathrm{~d}$, or lsminsc was utilized for the despeckling of symptomatic images in which the expert is fascinated in recognizing the degree of stenosis and the plaque borders.

An inherent property of medical ultrasound imaging is Speckle noise, and it generally tends to minimize the image resolution and contrast, thereby minimizing the diagnostic value of this imaging modality. A simple preprocessing procedure have been proposed by Oleg Michailovich et al. [50], which have customized the acquired radio-frequency images (without affecting the anatomical information they contain), in order that the noise in the log-transformation domain turned out to be similar in its behavior to a white Gaussian noise. As a result, based on assuming the noise to be white and Gaussian, the preprocessing has been allowed filtering methods, to perform in nearly optimal conditions. Nonlinear Filters Wavelet Denoising, Total Variation Filtering, and Anisotropic Diffusion are the three different performances that have been evaluated by the study and demonstrated that, the proposed preprocessing significantly have enhanced the quality of resultant images in all these cases. A series of computer-simulated and in vivo experiments have been comprised in their numerical tests.

A speckle reduction method, i.e., Laplacian Pyramid-based Nonlinear Diffusion (LPND), has been proposed by Fan Zhang et al. [51] for medical ultrasound imaging. In Laplacian pyramid domain by means of their method, speckle was detached by nonlinear diffusion filtering of band pass ultrasound images. A gradient threshold was automatically resolved by a variation of Median Absolute Deviation (MAD) estimator for nonlinear diffusion in each pyramid layer. With that of the other speckle reduction methods the performance of the proposed LPND method has been contrasted, as well as the Speckle Reducing Anisotropic Diffusion (SRAD) and Nonlinear Coherent Diffusion (NCD). In simulation and phantom studies, compared to SRAD the proposed LPND method could effectively preserved the edges and detailed structures while thoroughly suppressing speckle and NCD, an average gain of $1.55 \mathrm{~dB}$ and $1.34 \mathrm{~dB}$ in contrast-to-noise ratio was attained, respectively. The proposed LPND method could effectively preserved the edges and detailed structures while thoroughly suppressing speckle and this has been revealed by the visual comparison of despeckled in vivo ultrasound images from liver and carotid artery. Their preliminary results have indicated that the proposed speckle reduction method in medical ultrasound imaging, have improved the image quality and the visibility of small structures and fine details.

Image denoising has turned out to be a very essential implement all through diagnose in medical image processing. In medical images, Negotiation between the preservation of useful diagnostic information and noise suppression must be treasured. For instance in Ultrasound images, the noise have controlled the information which was valuable for the general practitioner in certain cases. The triumph of ultrasonic examination has depended on the Image quality. A particular type of acoustic noise, technically known as speckle noise, was the major factor of image quality degradation in case of ultrasonic images. For effective suppression of speckle noise, many denoising techniques have been proposed. Sudha et al. [52] have presented the performance analysis of a variety of schemes for suppressing speckle noise in Ultrasound images in terms of the assessment parameters PSNR and Equivalent Number of Looks (ENL).

In image processing, to progress the qualitative inspection of the image and the performance of quantitative image analysis techniques, restoration is anticipated. Pierrick Coupe et al. [53] have proposed an adaptation of the Non Local (NL-) means filter for speckle reduction in Ultra Sound (US) images. Formerly expanded for additive white Gaussian noise, to derive a NL-means filter adapted to a relevant ultrasound noise model they have proposed to exploit a Bayesian framework. Compared to well-established and state-of-the-art methods, the Quantitative results on synthetic data have shown that the performances of the proposed method. Consequences on real images have illustrated that the proposed method was capable to maintain edges and structural details of the image accurately.

Due to its noninvasive nature, low cost and capability of forming real time imaging, Ultrasound imaging is a widely exploited and safe medical diagnostic technique. However by the existence of signal dependant noise known as speckle, the utility of ultrasound imaging was degraded. On the structure of the image tissue and a variety of imaging parameters the speckle pattern has been depended. The two major causes for speckle reduction in medical ultrasound imaging are: (1) to progress the human interpretation of ultrasound images (2) despeckling was the preprocessing step for various ultrasound image processing tasks such as segmentation and registration. In ultrasound imaging, numerous methods have been proposed for speckle reduction. It has to be considered that certain speckle encloses diagnostic information and should be retained while incorporating speckle reduction techniques as a support for visual diagnosis. Kalaivani Narayanan et al. [55] have proposed an overview about types of speckle reduction techniques in ultrasound imaging.

Thangavel et al. [54] have proposed diverse filtering techniques based on statistical methods for the removal of speckle noise. The proposed filtering model has been validated using a number of successful experiments. By the statistical quantity measures: Signal-to-Noise Ratio (SNR), Peak Signal-to-Noise Ratio (PSNR), and Root Mean Square Error (RMSE) the quality of the enhanced images was measured. The computational outcome has showed that one of the proposed algorithms M3- 
Filter has executed in an enhanced manner than others.

In ultrasound medical images, an algorithm has been proposed and analyzed by Ratha Jeyalakshmi et al. [56] for cleaning speckle noise. In this algorithm, Mathematical Morphological operations were utilized. That algorithm was depended on Morphological Image Cleaning algorithm (MIC) designed by Richard Alan Peters II. A different technique has been handled by the algorithm, for redo the features that are lost while eliminating the noise. It has also used arbitrary structuring elements appropriate for the ultrasound images which comprise speckle noise for morphological operations.

Mariana Carmen Nicolae et al. [57] have proposed an ultrasound image enhancement algorithm based on the wavelet transform. It was hard to categorize speckle from the signal by only exploiting magnitude statistics of wavelet coefficients in the decomposed image. At each resolution scale, to discriminate speckle from the signal, they have acquired the structural information from the wavelet decomposed image. Later, on the basis of the structural information, they have applied the directional filtering and speckle reduction procedures adaptively to the multi-resolution image. The experimental consequences have exposed that without generating any noticeable artifact the proposed algorithm enhanced the subjective image quality considerably, and afforded better performance compared with the existing enhancement schemes. Also, using nonlinear processing of wavelet coefficients, they have done speckle noise removal. For an exact matching of the signal and noise distributions at various scales and orientations their algorithm was tested and found to be effective. Finally, they have noted that for the principle of denoising other types of biomedical images, their algorithm was easily adapted.

A simple and efficient algorithm for speckle noise reduction of the US B-Scan image has been proposed by Sivakumar et al. [58] which pooled the Wiener filtering and thresholding methods in the wavelet transform domain. To review the performance of wavelet thresholding techniques viz., VisuShrink, BayesShrink and SureShrink, experiments were conducted on the three different ultrasound images corrupted by speckle noise. In addition, among the Wiener filter with Wavelet thresholding and other standard filters like Frost, Lee, Kuan, Median and Wiener filters a comparative study has been made. The upshot of their study revealed that in terms of RMSE, PSNR and visual quality, Wiener filter with BayesShrink thresholding scheme has outperformed all other filters.

Based on the hybridization of wavelet and bilateral filters, Sudipta Roy et al. [59] have proposed a model for denoising of variety of noisy images. The model was experimented on standard images, approximating x-ray images, ultrasound and astronomical telescopic images and the performances were evaluated in terms of Peak Signal to Noise Ratio (PSNR) and Image Quality Index (IQI). Results have demonstrated that the utilization of bilateral filters in combination with wavelet thresholding filters on sub bands of a decomposed image has depreciated the performance. But the application of bilateral filter has enhanced the performance, before and after decomposition. Amongst four models considered in their study in terms of PSNR and IQI, the filter progressed with bilateral filter before and after the decomposition of an image was found to be the best performance. In addition, nearly uniform and consistent results on all the images have been given by the proposed filter.

Due to the poor contrast, weak texture structure, the speckle noise, and shadow regions, Prostate texture analysis is a very tricky and challenging task. Hence the standard and conventional image processing methods of the prostate region are not capable of segmentation. An adaptive anisotropic diffusion method has been proposed by Nezamoddin Kachouie to progress the selective within-region image smoothness while preserving the region boundaries [60]. They have widened the anisotropic diffusion to numerous directions such that on the basis of rich extracted features, the segmentation methods can be applied efficiently. Based on extended diffusion, a preliminary segmentation method was proposed. It was widened to eight directions, i.e., four directional pairs such that diffusion in each direction was separately calculated yielding a reach feature set for ultrasound image enhancement and analysis.

\subsection{Computed Tomography (CT) Image}

Jou-Wei Lin et al. [61] have provided the utilization of multiscale wavelet transforms on image-based physiological quantification and provided significant information for clinical application. They have illustrated that non invasive quantification with $\mathrm{Rb}$ accurately measures myocardial perfusion in human subjects by using dynamic PET images. In clinical applications, to quantify a variety of physiological or pathological processes, the approach has been able to generalize to other dynamic image modalities with similar tracers or contrast media. The estimation of functional parameters has been improved in a wide array of spatial/temporal imaging modalities in medicine by clinical use of those methods.

Using Discrete Wavelet Transform (DWT), Arivazhagan et al. [13] has experimentally analyzed the performance of an Image Denoising System for four levels of DWT decomposition, i.e., Speckle noise added two facial and two CT images. From their comprehensive experiments conducted with the expanded image denoising software for diverse noise parameters and for diverse levels of DWT decomposition by exploiting soft thresholding technique, the following conclusions were derived: (i) The maximum PSNR $(\mathrm{dB})$ was attained for first level of DWT decomposition (SNR1) for most of the images added with Speckle noise, (ii) In addition, it was observed that the SNR attained for superior level of DWT decomposition was lesser than SNR1 or SNR2 and for the fourth level of DWT decomposition, severe blurring occurs, irrespective of images and noise parameters and (iii) In addition, it was interested to notice that for images corrupted with lesser noise densities, single level of DWT decomposition was sufficient; while for images corrupted with higher noise densities irrespective of images second level of DWT decomposition was required.

Using Dental tomographic cone beam X-ray imaging devices the truncated projections are recorded and a region of interest (ROI) has been reconstructed within the head. An ill-posed inverse problem was the Image reconstruction from the local tomography data. Niinimaki et al. [62] has proposed a Bayesian multi-resolution method for the local tomography reconstruction. In a well-posed statistical form, the inverse problem was formulated where a prior model of the target tissues was remunerated for the incomplete X-ray projection data. Tissues are symbolized in a wavelet basis, and in terms of Besov norm penalty, the prior information was modeled. By abandoning fine-scale wavelets outside the ROI, the number of unknowns in the reconstruction problem was minimized. Significant reduction of degrees of freedom has been allowed by the proposed multi resolution approach, without loss of accuracy inside the ROI by using simulated and in vitro local tomography data.

Due to the occurrence of noise, reconstructing low-dose 
Computed Tomography (CT) images was an unstable inverse problem. To address that problem, Thavavel et al. [63] have proposed a regularized reconstruction method that merges the features from the Filtered Back-Projection (FBP) algorithm and regularization theory. In complex wavelet domain, the filtering part of FBP has been comprised of Fourier-domain inversion followed by noise suppression based on thresholding procedure. To eliminate blurring and noise without the need for assuming a specific noise mode the proposed method have exploited the properties of dual tree complex wavelet transform (DT-CWT). In addition, to suppress the noise while preserving the sharpness of the reconstructed image, it has used an adaptive shrinkage function based on median, mean and standard deviation of wavelet coefficients. With the projections simulated from Shepp-Logan Phantom, the efficacy of the proposed method was assessed. Simulation results have confirmed that in terms of suppressing noise and preserving resolution in the reconstructed images, the proposed method has produced consistently good reconstruction.

Ali et al. [5] have proposed a curvelet based approach for the fusion of Magnetic Resonance (MR) and Computed Tomography (CT) images. Between the traditional wavelet fusion algorithm and the proposed curvelet fusion algorithm, a comparison study has been prepared. The experimental study has shown that to the application of the traditional wavelet transform, the application of the curvelet transform in the fusion of MR and CT images was superior. The obtained curvelet fusion results have elevated PSNR values than the wavelet fusion results. Also, than in the wavelet fusion results, curved visual details were enhanced in the curvelet fusion outcomes.

In medical and biological imaging, Multiplicative noise occurs. In medical images, Noise reduction was a tricky task in which linear filtering algorithms fails forever. Bayesian algorithms were exploited with accomplishment but they were time consuming and computationally demanding. In addition, in medical diagnosis procedures, the mounting significance of the 3-D and 4-D medical image analysis has augmented the amount of data that must be efficiently processed. Joao Sanches et al. [64] have proposed a Bayesian denoising algorithm which has handled with additive white Gaussian and multiplicative noise described by Poisson and Rayleigh distributions. The algorithm was based on the Maximum a Posteriori (MAP) criterion, and edge preserved priors which have shunned the distortion of relevant anatomical details. The amalgamation of a set of Bayesian denoising algorithms was the main contribution of them, for additive and multiplicative noise using a well-known mathematical framework, the Sylvester-Lyapunov equation, developed in the context of the Control theory.

Fast Discrete Curvelet Transform has been implemented; analyzed and compared by Kiran Kumar et al. [65] using Wrapper algorithm based image fusion technique with Wavelet based Fusion Technique. The task has intended at fusion of two images comprising varied information. In a single pass, the proposed algorithm has been taken care of registration as well as fusion. To fuse MRI with CT and MR/MR images of Preclinical data, attempt has been taken. Three bands of images ("MRI triplet") are available in magnetic resonance imaging (MRI), which is T1-, T2- and PD-weighted images. Complementary structure information has been provided by three images of a MRI triplet and therefore it was supportive for diagnosis and subsequent analysis to merge three band images into one. Significantly medical diagnosis and also the further image processing such as, visualization (colorization), segmentation, classification and Computer-Aided Diagnosis (CAD) are benefited by that fused images. By utilizing quantitative fusion metrics such as the Entropy, Difference Entropy, and Standard Deviation, Image Quality Index (IQI) and ratio Spatial Frequency error (rSFe), the approach was then optimized.

In distinguishing the broad range of gray level values, Human vision system possesses limitations. Pixel intensities up to 15 30 gray levels can be discriminated by human eye. The qualitative analysis of radiological images is restricted by this. Hence to reveal more information from the image, quantitative analysis is preferred. Ratnaparkhe et al. [66] have proposed the Texture feature extractions based on Ridgelet transform. In the initial step of their effort, the texture features from Region of Interest (ROI) have been determined. The texture features have been represented by the energy and entropy in partitions of Ridgelet transform images. Two-class and multi-class classification have been carried out in the second step of their effort. They have reported the Percentage Correct Classification for Ridgelet based energy and entropy features and comparative analysis of performance measures for diverse organ images.

To estimate noise and remove it from digital images, in order to achieve a good performance Aliaa Youssif et al. [67] have proposed an adaptive threshold method for image denoising based on curvelet transform. In estimation, the proposed adaptive threshold method has been more efficient and reduces noise from images which have random, salt \& pepper and Gaussian noise. Experimental results have shown that an enhanced denoising performance over related earlier techniques has been demonstrated by the proposed method according to increasing of PSNR values of enhanced images by 0.044 at Random, 1.05 at salt \& pepper and 0.457 at Gaussian noise.

A Computer-Aided Diagnostic system has been proposed by Kumar et al. [68] for the diagnosis of benign and malignant liver tumors from Computed Tomography (CT) images by using curvelet transform based multi-resolution texture feature extraction and neural network. Hepatocellular carcinoma and cavernous hemangioma are the two kinds of identified liver diseases. The experiment outcomes have revealed that the classification accuracy of curvelet based diagnosis was higher than the wavelet based method. By increasing the number of samples, the performance was increased more. For diagnosis of other types of liver diseases, the proposed system can be extended.

In image compression and/or encoding/decoding of Region of Interest (ROI), most of the commercial medical image viewers do not afford scalability. A medical application has been proposed by Lalitha et al. [69] that encloses a viewer for Digital Imaging and Communications in Medicine (DICOM) images as a core module. Scalable wavelet-based compression, retrieval, and decompression of DICOM medical images have been enabled by the proposed application and also supported the ROI coding/decoding. In addition, by activating mobile devices in a heterogeneous network, the presented application was appropriated for utilization. The methodology have concerned extracting a known DICOM image into two segments, compressing the region of interest with a lossless, quality sustaining compression scheme like JPEG2000, compressing the insignificant regions ( background, et al.,) with an algorithm that has a very high compression ratio and that did not focused on quality (SPIHT). Energy efficiency was achieved with that type of the compression work, and the outputs were integrated and combined with the output from a texture based edge detector after respective reconstructions. Hence the essential targets were achieved and texture information was maintained.

The process of partitioning voxels into 3D regions (sub 
volumes) that symbolize significant physical entities which are more significant and easier to analyze and exploitable in future applications is termed as $3 \mathrm{D}$ volume segmentation. According to certain levels of resolution or blurring, Multi-Resolution Analysis (MRA) has been enabled the preservation of an image. Wavelets have been deployed in image compression, denoising, and classification, because of multi-resolution quality. Shadi Al Zubi et al. [70] have proposed the implementation of efficient medical volume segmentation techniques. For feature extraction, Multi-resolution analysis as well as 3D wavelet and ridge let have been exploited which can be modeled using Hidden Markov Models (HMMs) to segment the volume slices. To evaluate 2D and 3D techniques, a comparison study has been carried out which have revealed that 3D methodologies detect the Region of Interest (ROI) accurately. Using HMMs, Automatic segmentation has been achieved where the ROI was detected accurately but for its calculations, it undergoes a long computation time.

A process that converts from one resolution to another without losing the visual content in the photo is termed as Image interpolation. Physician needs to zoom in on specific parts of CT scanned image in medical imaging. To observe the detailed information and to determine obviously, the image needs to enlarge image size. They can be degraded due to blocky and jaggy effects when the small size images/portions were enlarged. By interpolation process, this process must be followed. This is the challenge to acquire sharp or obvious image from these processes. Numerous interpolation methods are instigated. However, most photo editing software can not widen the result images with obvious information. To restore or sharp blurred images due to zooming or magnification process and to aid in medical analysis is the major contribution and it has been proposed by Ah Nge Htwe [71]. He has exploited NL (non-local) means interpolation method which can recover texture well. For medical imaging, he has also mentioned the experiment how their process was efficient and generates high quality images.

\subsection{Medical Resonance Imaging (MRI)}

A wavelet domain method has been proposed by Aleksandra Pizurica et al. [72] for noise filtering in medical images. Their method adapted itself to a variety of sorts of image noise as well as to the preference of the medical expert: a single parameter can be utilized to equilibrium the preservation of relevant details against the degree of noise reduction. Generally, valid knowledge about the correlation of significant image features across the resolution scales has been exploited by their algorithm to carry out a preliminary coefficient classification. To empirically estimate the statistical distributions of the coefficients that represent useful image features on the one hand and mainly noise on the other, the preliminary coefficient classification was used. By using a wavelet domain indicator of the local spatial activity, the adaptation to the spatial context in the image was achieved. Both in its implementation and execution time their proposed method was of low-complexity. For noise suppression in medical ultrasound and magnetic resonance imaging, their results have demonstrated its efficacy. In its applications, in terms of quantitative performance measures as well as in terms of visual quality of the images, the proposed method obviously outperforms single-resolution spatially adaptive algorithms.

MRI image denoising scheme has been proposed by Paul Bao et al. [73] using an adaptive wavelet thresholding technique. Unlike many traditional schemes that directly threshold the wavelet coefficients, to amplify the significant features, their scheme multiplies the adjacent wavelet sub bands and then to differentiate edge structures in a better manner from noise it applies the thresholding to the multi-scale products. To eliminate the majority of the noise the distribution of the products was analyzed and an adaptive threshold was formulated. Experiments results of MRI images have exposed that the proposed scheme not only achieved high MSR and CNR measurements but also conserved additional edge features.

Lei Jiang et al. [74] have proposed an adaptive wavelet-based Magnetic Resonance images denoising algorithm. A Rician distribution for background-noise modeling was introduced and for the parameter estimation procedure a Maximum-Likelihood method has been employed. In addition by updating the shrinkage function along consecutive scales and applying spatial constraints, discrimination between edge and noiserelated coefficients was achieved. On both simulated and real Magnetic Resonance images, the efficiency of the algorithm was demonstrated. The results have revealed to be capable and outperformed other denoising approaches.

McGraw et al. [75] have discussed that diffusion tensor imaging could give the essential information necessary for viewing structural connectivity. Nevertheless, robust and precise acquisition and processing algorithms have been required to map the nerve connectivity precisely. They have presented an algorithm for extracting and visualizing the fiber tracts in the CNS, exclusively in the brain. The automatic fiber tract mapping problem has been solved in two stages, namely a data smoothing stage and a fiber tract mapping stage. In the former, smoothing of the diffusion-weighted data (prior to tensor calculation) has been attained by means of a weighted TV-norm minimization, which has attempted to smooth while preserving all significant details. For the fiber tract mapping, a smooth 3D vector field representing the dominant anisotropic direction at every spatial location has been calculated from the smoothed data. Neuronal fibers have been then located by evaluating the integral curves of this vector field. Outcomes have been illustrated using three modes of visualization: (1) Line integral convolution has given an oriented texture which has shown fiber pathways in a planar slice of the data. (2) A stream tube map has been produced to present a 3D view of fiber tracts. Additional information, for instance degree of anisotropy, could be encoded in the tube radius, or by using color. (3) A particle system form of visualization has also been offered. This mode of display has been permitted for interactive exploration of fiber connectivity with no supplementary preprocessing.

Yasser M. Kadah [76] has proposed an adaptive signalpreserving technique based on spectral subtraction for noise elimination in event-related functional magnetic resonance imaging (fMRI) data. The proposed technique has estimated a parametric model for the power spectrum of random noise from the attained data based on the characteristics of the Rician statistical model. The proposed model has been later utilized to compute a noise-suppressed power spectrum for any given pixel time course by simple subtraction of the power spectra. The proposed technique has been tested using computer simulations and real data from event-related fMRI experiments. The results have illustrated the potential of the method in suppressing noise while retaining the other deterministic components in the signal. Also, further analysis using principal component analysis and independent component analysis have demonstrated a significant enhancement in both convergence and clarity of results when the technique is utilized. Given its simple form, the new method has neither modified the statistical characteristics of the signal nor caused correlated noise to be 
present in the processed signal. Thus, the value of the proposed technique as a useful preprocessing step for fMRI data analysis has been demonstrated.

Based on a combination of the total variation minimization scheme and the wavelet scheme a denoising algorithm for medical images has been proposed by YangWang et al. [77]. While maintaining sharpness of objects, they have showed that their scheme offers effective noise removal in real noisy medical images. Further, their scheme permits us to implement an effective automatic stopping time criterion significantly.

Nicolas Wiest Daessle et al. [78] have proposed a technique to adapt the NL Means filter to Rician noise corrupted data. For both conventional MR images and DT images, Validation was performed on synthetic data and on real data. For DW-MRI, their adaptation outperformed the original NL Means filter in terms of Peak-Signal-to-Noise Ratio (PSNR).

A dynamic thresholding and image normalization technique has been proposed by Nur Faiza Ishak et al. [15] for enhancement of low-field brain MRI images. By controlled truncating and normalizing the histogram, the surplus background noise and artifacts in the images were eliminated by their scheme. While approximately no loss of information or image integrity was recorded in the actual brain areas of the processed low-field MRI images, the clarity of the ROI was enhanced effectively. In their paper the histogram of a typical low-field MRI image, the first peak is inclined to be the image background, with the second peak being the acquisition artifact. The intensity around the third peak was the part of concern, which has been consisted of the major brain regions. As qualitative visual human observation may be subjective, quantitative measurement had also been taken into consideration to estimate the differences between the original and preprocessed images. Minor inaccuracy had been detected out of entire tested database. Hence, the results shows that more than $85 \%$ of the test images have been performed as expected in eliminating all nonROIparts.

Andre Souza et al. [79] have discussed that in medical imaging, low signal-to-noise ratio (SNR) and/or contrast-to-noise ratio (CNR) have frequently caused several image processing algorithms to perform defectively. Postacquisition image filtering has been a significant off-line image processing approach extensively used to improve the SNR and CNR. Image degradation by diffusing/blurring edges and/or fine structures has been a significant drawback in several filtering techniques. They have introduced a scale-based filtering method that utilizes scale-dependent diffusion conductance to perform filtering. Their proposed approach has employed object scale information by means of a concept called generalized scale, which does not necessitate shape, size, or anisotropic constraints unlike ball scale-based filtering approaches. The object scale has permitted them to control the filtering process by limiting smoothing to regions with fine details and in the neighborhood of boundaries while allowing effective smoothing in the interior of homogeneous regions. A quantitative evaluation strategy that captures the SNR to CNR trade-off behavior of filtering methods has been presented. The evaluations based on the Brainweb data sets have demonstrated the superior performance of the generalized scale-based diffusive filtering over the existing methods, namely, ball scalebased and nonlinear complex diffusion processes. A qualitative experiment which is based on both phantom and patient magnetic resonance images has demonstrated that the generalized scale-based approach leads to better retention of fine details and edges.
Normally, Magnetic Resonance images are corrupted by random noise from the measurement process complicating the automatic feature extraction and analysis of clinical data. It was because of denoising methods that have been traditionally applied to progress MR image quality. A filter has been proposed by Jose Manjon et al. [80] to minimize random noise in multi-component MR images by spatially averaging similar pixels using information from all available image components to perform the denoising process. As a post processing step, their proposed algorithm have exploited a local Principal Component Analysis decomposition to eliminate additional noise without significantly affecting the original image resolution, by exploiting information not only in the spatial domain but also in the inter-component domain dealing in a higher noise reduction. With similar state-of-art methods, the proposed method has been compared over synthetic and real clinical multi-component MR images showing an improved performance in all cases analyzed.

Implementations of denoising algorithms have demonstrated by Jaya et al. [81] on MR brain images. In denoising MR brain images, a major concern was the poor quality images secondary to a worsening Signal to Noise Ratio (SNR). On the application of preprocessing techniques towards segmenting and labeling the brain images, their paper gave some useful insight. Confidential results are reported. Their Proposed technique comprises four processing stages. The MRI brain image was acquired from MRI brain data set to MATLAB in the initial stage. The MRI was fed to the pre-processing stage after acquisition, the film artifacts (labels) were removed at this stage. In the third stage, using 1.Median filter 2.Weighted median 3.Adaptive filter, the high frequency components and noise were eliminated from MRI. Finally, the performance of above filters were measured and evaluated.

Rajeesh et al. [82] has been proposed the denoising of Magnetic Resonance Images using wave atom shrinkage. They have proved that compared to wavelet and curvelet shrinkages the proposed approach had achieved a better SNR. A precise advantage of their proposed method is the edge preserving property. Moreover, the efficiency of proposed method will be accentuated by comprising a large dataset of real-time normal and pathological MR images.

A super-resolution has been proposed by Jose Manjon et al. [83] method to recover high frequency information lost using coplanar high resolution images. Their proposed methodology has gained benefit from the fact that in typical clinical settings both high and low-resolution images of different types were taken from the similar subject. To progress the resolution of other coplanar lower resolution images effectively, those available high resolution images were employed. To illustrate the effectiveness of the proposed approach, experiments on synthetic and real data were supplied. To demonstrate the enhanced performance of that proposed methodology over previous State-of-the-art methods, a comparison with classical interpolation techniques was presented.

\section{DIRECTIONS FOR THE FUTURE RESEARCH}

In this review paper, different methods developed for denoising the medical images are thoroughly analyzed. Analysis has been done on the Radiography, Ultrasound, MRI and CT images. Besides others, the $\mathrm{CT}$ image plays a more important role because it is one of the most common and very significant modalities employed in medical imaging. Hence due to its prevalent utilization, obtaining better results is essential for CT images. This paper will be a healthier foundation for the 
budding researchers in identifying appropriate denoising techniques for medical images and especially for CT images. In future we expect numerous brainwaves will rise by means of our review work.

\section{CONCLUSION}

Medical image denoising is an emergent research area that has received great attention among the researchers in recent years. In this paper, a broad review of the significant researches and techniques that exist for medical image denoising is pursued. Here the researches are first categorized into Radiographic, Ultrasound, MRI and CT images based on the type of the medical image. Then, followed by a concise description on digital images and medical images and a brief discussion about each category of medical images the salient features of the important researches existing in the literature are reviewed. Thus, this review paves the path for the budding researchers to get acquainted with the various techniques existing in the denoising process of medical images.

\section{REFERENCES}

[1] Kevin Chiu, Tejas Nadkarni, Swati Kumar and Avanti Dharkar, "EZIP: Easy Image Processing", Technical Report, 2007

[2] Carl Steven Rapp, "Image Processing and Image Enhancement", Frontiers in Physiology, 1996

[3] John Russ, "The Image Processing Handbook", Library of Congress Cataloging-in-Publication Data, Vol.3, 1999

[4] Asma Yasrib and Mohd Adam Suhaimi, "Image Processing in Medical Applications", Journal of Information Technology Impact, Vol. 3, No. 2, pp.63-68, 2003

[5] Ali, El-Dokany, Saad and Abd El-Samie, "Curvelet Fusion of MR and CT Images", Progress In Electromagnetics Research, Vol. 3, pp.215-224, 2008

[6] Masroor Ahmed and Dzulkifli Bin Mohamad, "Segmentation of Brain MR Images for Tumor Extraction by Combining K-means Clustering and Perona-Malik Anisotropic Diffusion Model" International Journal of Image Processing, Vol.2, No.1, pp.27-34, 2008

[7] George Karkavitsas and Maria Rangoussi, "Object localization in medical images using genetic algorithms", World Academy of Science, Engineering and Technology, 2005

[8] John Stoitsis, Ioannis Valavanis, Stavroula Mougiakakou, Spyretta Golemati, Alexandra Nikita and Konstantina Nikita, "Computer aided diagnosis based on medical image processing and artificial intelligence methods", Nuclear Instruments and Methods in Physics Research, Vol.569, No.2, pp.591-595, 2006

[9] De Souza, De Azevedo and Marino-Neto, "Didactic Platform for Biomedical Signal Processing: Digital Image Processing",

[10] Anca Morar, Florica Moldoveanu, Alin Moldoveanu, Victor Asavei And Alexandru Egner, "Medical Image Processing in Hip Arthroplasty", Wseas Transactions On Signal Processing, 2010

[11] Syed Amjad Ali, Srinivasan Vathsal and Lal kishore, "CT Image Denoising Technique using GA aided Windowbased Multiwavelet Transformation and Thresholding with the Incorporation of an Effective Quality Enhancement Method", International Journal of Digital Content Technology and its Applications, Vol.4, No. 4, 2010
[12] Yang Wang And Haomin Zhou, "Total Variation Wavelet Based Medical Image Denoising", International Journal of Biomedical Imaging, Vol. 2006,

[13] Arivazhagan, Deivalakshmi and Kannan, "Performance Analysis of Image Denoising System for different levels of Wavelet decomposition", International Journal of Imaging Science and Engineering, Vol.3, 2007

[14] Syed Amjad Ali, Srinivasan Vathsal and Lal kishore, "A GA-based Window Selection Methodology to Enhance Window-based Multi-wavelet transformation and thresholding aided CT image denoising technique", (IJCSIS) International Journal of Computer Science and Information Security, Vol. 7, No. 2, 2010

[15] Nur Faiza Ishak, Rajasvaran Logeswaran, Wooi-Haw Tan, "Artifact and noise stripping on low-field brain MRI", International Journal Of Biology And Biomedical Engineering, Vol.2, No.2, 2008

[16] Sivakumar, Denoising Of Computer Tomography Images Using Curvelet Transform, ARPN Journal of Engineering and Applied Sciences, Vol. 2, No.1, 2007

[17] Pierrick Coupé ,Pierre Yger, Sylvain Prima, Pierre Hellier, Charles Kervrann , Christian Barillot, "An optimized blockwise nonlocal means denoising filter for 3-D magnetic resonance images", Vol.27, No.4, pp.425-441, 2008

[18] Mona MKA Youssef Booz and FFR RCSI, "Advances in Radiology", Bahrain Medical Bulletin, Vol.32, No.1, 2010

[19] Lu,Nickoloff,So and Dutta, "Comparison of computed radiography and film screen combination using a contrastdetail phantom", Journal Of Applied Clinical Medical Physics, Vol.4, No.1, 2003

[20] Mark Williams,Elizabeth Krupinski,Keith Strauss, William Breeden,Mark Rzeszotarski, Kimberly Applegate, Margaret Wyattg, Sandra Bjork and Anthony Seibert, "Digital Radiography Image Quality: Image Acquisition",Vol.4,pp.371-388. 2007

[21] Michiel Schaap, Arnold Schilham, Karel Zuiderveld, Mathias Prokop, Evert-Jan Vonken and Wiro Niessen,"Fast Noise Reduction in Computed Tomography for Improved 3-D Visualization", Ieee Transactions On Medical Imaging, Vol.27, No.8,2008

[22] Kwan-Hoong, Azlan Ahmad, Nizam and Abdullah, "Magnetic Resonance Imaging: Health Effects and Safety", Proceedings of the International Conference on Non-Ionizing Radiation at UNITEN, Electromagnetic Fields and Our Health, 2003

[23] Kulkarni, Meher and Nair, "An Algorithm for Image Denoising by Robust Estimator", European Journal of Scientific Research, Vol.39, No.3, pp.372-380, 2010.

[24] Radu Ciprian Bilcu and Markku Vehvilainen, "A NOVEL DECOMPOSITION SCHEME FOR IMAGE DENOISING", IEEE International Conference on Acoustics, Speech and Signal Processing, pp.577-580, 2007.

[25] K. Arulmozhi, S. Arumuga Perumal, K. Kannan, S. Bharathi, "Contrast Improvement of Radiographic Images in Spatial Domain by Edge Preserving Filters", IJCSNS International Journal of Computer Science and Network Security, VOL.10 No.2, February 2010.

[26] G. M. Atiqur Rahaman and Md. Mobarak Hossain," Automatic Defect Detection and Classification Technique From Image: A Special Case Using Ceramic Tiles", 
(IJCSIS) International Journal of Computer Science and Information Security, Vol. 1, No. 1, May 2009

[27] Hiroyuki Takeda, Sina Farsiu and Peyman Milanfar, "Kernel Regression for Image Processing and Reconstruction”, IEEE Transactions on Image Processing, VOL. 16, NO. 2, Feb 2007.

[28] Peter Kovesi, "Phase Preserving Denoising of Images", 2007

[29] Lakhwinder Kaur, Savita Gupta and Chauhan, "Image Denoising using Wavelet Thresholding", Indian Conference on Computer Vision, Graphics and Image Processing, 2002

[30] Yong-Hwan Lee and Sang-Burm Rhee, "Wavelet-based Image Denoising with Optimal Filter", International Journal of Information Processing Systems, Vol.1, No.1, 2005

[31] Geoff Dougherty, "Digital Image Processing for Medical Applications", Cambridge University Press, 2009

[32] Yauso Suga, Kouichiro Kojima and Tetsuro Tominaga, "Detection of Weld Defects by Computer-Aided X-Ray Radiography Image Processing", International Journal of Offshore and Polar Engineering, Vol. 5, No.2, 1995

[33] Elizabeth Krupinski, Mark Williams, Katherine Andriole, Keith Strauss, Kimberly Applegate, Margaret Wyattf, Sandra Bjork and Anthony Seibert, "Digital Radiography Image Quality: Image Processing and Display", Journal of the American College of Radiology, Vol. 4, No. 6, 2007

[34] Karimi, Moghaddam, Naematollahi And Rezazadeh, "In Vitro Ultrasonography Of The Normal Sheep Heart", Pakistan Vet. J., No.28, No.2, pp.92-94, 2008

[35] Eze, "Pattern of Computed Tomography Findings in Cerebrovascular Accident Patients in South-Eastern Nigeria - A Retrospective Study of 480 Patients", European Journal of Scientific Research, Vol.34 No.1, pp.104-109, 2009

[36] William Scarfe, FRACDS, Allan Farman and Predag Sukovic, "Clinical Applications of Cone-Beam Computed Tomography in Dental Practice", JCDA, Vol. 72, No.1, 2006

[37] Martin Stahl, Til Aach and Sabine Dippel, "Digital Radiography Enhancement by Nonlinear Multiscale Processing", Medical Physics, Vol. 27, No.1, pp.56-65, 2000.

[38] Sabine Dippel, Martin Stahl, Rafael Wiemker, and Thomas Blaffert, "Multiscale Contrast Enhancement for Radiographies: Laplacian Pyramid Versus Fast Wavelet Transform", IEEE Transactions On Medical Imaging, Vol.21, No.4, pp.343-353, 2002.

[39] Hakan Oktem, Karen Egiazarian, Jarkko Niittylahti and Juha Lemmetti, "An Approach to Adaptive Enhancement of Diagnostic X-Ray Images", EURASIP Journal on Applied Signal Processing, Vol. 2003, No. 5, pp.430-436, 2003.

[40] Giakos, Fraiwan, Patnekar, Sumrain, Mertzios and Periyathamby, "A Sensitive Optical Polarimetric Imaging of Aircraft Turbine Engines Technique for Surface Defects Detection", IEEE Transactions On Instrumentation And Measurement,Vol.53, No.1, pp.216-222, 2004.

[41] Pierre Gravel, Gilles Beaudoin and Jacques De Guise, "A Method for Modeling Noise in Medical Images", IEEE
Transactions On Medical Imaging, Vol. 23, No. 10, pp.1221-1232, 2004.

[42] Mark Winslow, George Xua and Birsen Yazici, "Development of a simulator for radiographic image optimization", Computer Methods and Programs in Biomedicine, Vol.78, No.3, pp.179-190, 2005

[43] Triet Le, Rick Chartrand and Thomas Asaki, "A Variational Approach to Reconstructing Images Corrupted by Poisson Noise", Journal of Mathematical Imaging and Vision, Vol.27, No.3, pp.257-263, 2007.

[44] Frosio and Borghese, "Statistical Based Impulsive Noise Removal in Digital Radiography", IEEE Transactions On Medical Imaging, Vol. 28, No. 1, pp.3-16, 2009.

[45] Krishnamoorthy, Amudhavalli and Sivakkolunthu, "An Adaptive Mammographic Image Enhancement in Orthogonal Polynomials Domain", International Journal of Computer and Information Engineering, Vol.4, No.2, pp.120-128, 2010.

[46] Su Cheol Kang and Seung Hong Hong, "Experimental and Theoretical Analysis of Wavelet-Based Denoising Filter for Echocardiographic Images", Studies in Health Technology and Informatics, Vol.84, No.2, pp.906-909, 2001

[47] Suiren Wan, Balasundar Raju, Mandayam Srinivasan, "Robust Deconvolution of High-Frequency Ultrasound Images Using Higher-Order Spectral Analysis and Wavelets", IEEE Transactions On Ultrasonics: Ferroelectrics And Frequency Control, Vol.50, No.10, pp.1286-1295, 2003

[48] Shujun Fu, Qiuqi Ruan, Wenqia Wang and Yu Li, "Feature Preserving Nonlinear Diffusion for Ultrasonic Image Denoising and Edge Enhancement", World Academy of Science, Engineering and Technology, Vol.37, No.2, pp.148-151, 2005

[49] Christos Loizou, Constantinos Pattichis, Christodoulos Christodoulou, Robert Istepanian, Marios Pantziaris and Andrew Nicolaides, "Comparative Evaluation of Despeckle Filtering In Ultrasound Imaging of the Carotid Artery", IEEE Transactions On Ultrasonics, Ferroelectrics, And Frequency Control, Vol.52, No.10, pp.1653-1669, 2005

[50] Oleg Michailovich and Allen Tannenbaum, "Despeckling of Medical Ultrasound Images",ieee transactions on ultrasonics, ferroelectrics, and frequency control, Vol.53, No.1, pp.64-78, 2006

[51] Fan Zhang, Yang Mo Yoo, Liang Mong Koh, and Yongmin Kim, "Nonlinear Diffusion in Laplacian Pyramid Domain for Ultrasonic Speckle Reduction", IEEE Transactions On Medical Imaging, Vol.26, No.2, pp.200211, 2007

[52] Sudha, Suresh and Sukanesh, "Comparative Study on Speckle Noise Suppression Techniques for Ultrasound Images", International Journal of Engineering and Technology, Vol.1, No.1, pp.57-62, 2009

[53] Pierrick Coupe, Pierre Hellier, Charles Kervrann and Christian Barillot, "NonLocal Means-based Speckle Filtering for Ultrasound Images", IEEE Transactions on Image Processing, Vol.18, No.10, pp. 2221-2229, 2009

[54] Thangavel, Manavalan and Laurence Aroquiaraj, "Removal of Speckle Noise from Ultrasound Medical Image based on Special Filters: Comparative Study", 
ICGST International Journal on Graphics, Vision and Image Processing (GVIP), Vol.9, No.3, pp.25-32, 2009

[55] Kalaivani Narayanan and Wahidabanu, "A View on Despeckling in Ultrasound Imaging", International Journal of Signal Processing, Image Processing and Pattern Recognition, Vol.2, No.3, pp.85-98, 2009

[56] Ratha Jeyalakshmi and Ramar, "A Modified Method for Speckle Noise Removal in Ultrasound Medical Images", International Journal of Computer and Electrical Engineering, Vol.2, No.1, pp.54-58, 2010

[57] Mariana Carmen Nicolae, Luminița Moraru and Laura Onose, "Comparative Approach For Speckle Reduction In Medical Ultrasound Images", Romanian Journal of Biophysics, Vol.20, No.1, pp.13-21, 2010

[58] Sivakumar and Nedumaran, "Comparative study of Speckle Noise Reduction of Ultrasound B-scan Images in Matrix Laboratory Environment", International Journal of Computer Applications, Vol.10, No.9, pp.46-50, 2010

[59] Sudipta Roy, Nidul Sinha and Asoke Sen, "A New Hybrid Image Denoising Method", International Journal of Information Technology and Knowledge Management, Vol.2, No.2, pp.491-497, 2010

[60] Nezamoddin Kachouie, "Anisotropic Diffusion for Medical Image Enhancement", International Journal of Image Processing (IJIP), Vol.4, No.4, 2010

[61] Jou-Wei Lin, Andrew Laine and Steven Bergmann, "Improving PET-Based Physiological Quantification Through Methods of Wavelet Denoising", IEEE Transactions On Biomedical Engineering, Vol.48, No.2,pp.202-212, 2001

[62]Niinimaki, Siltanen and Kolehmainen, "Bayesian multiresolution method for local tomography in dental Xray imaging", Physics in Medicine and Biology, Vol.52, No.22, pp.1-17, 2007

[63] Thavavel and Murugesan, "Regularized Computed Tomography using Complex Wavelets", International Journal of Magnetic Resonance Imaging, Vol.1, No. 1, pp. 27-32, 2007

[64]Joao Sanches, Jacinto Nascimento and Jorge Marques, "Medical Image Noise Reduction Using the SylvesterLyapunov Equation", IEEE Transactions On Image Processing, Vol. 17, No. 9, pp.1522-1539, 2008

[65] Kiran Kumar, "Comparison Of Fusion Techniques Applied To Preclinical Images: Fast Discrete Curvelet Transform Using Wrapping Technique \& Wavelet Transform", Journal of Theoretical and Applied Information Technology, Vol.5, No.6, pp. 668-673, 2009

[66] Ratnaparkhe, Manthalkar and Joshi, "Texture Characterization of CT Images Based on Ridgelet Transform", ICGST International Journal on Graphics, Vision and Image Processing (GVIP), Vol. 8, No.V, pp. 43-50, 2009

[67] Aliaa Youssif, Darwish and Madbouly, "Adaptive Algorithm for Image Denoising Based on Curvelet Threshold", International Journal of Computer Science and Network Security, Vol.10, No.1, pp. 322-328, 2010

[68] Kumar and Moni, "Diagnosis of Liver Tumor from CT Images using Curvelet Transform", International Journal on Computer Science and Engineering, Vol.2, No.4, pp.1173-1178, 2010
[69] Lalitha and Latte, "Lossless and Lossy Compression of DICOM images With Scalable ROI", International Journal of Computer Science and Network Security, Vol.10, No.7, pp.276-281, 2010

[70] Shadi AlZubi and Abbes Amira, "3DMedical Volume Segmentation Using Hybrid Multi resolution Statistical Approaches", Advances in Artificial Intelligence, Vol.2010, pp. 1-15, 2010

[71] Ah Nge Htwe "Image Interpolation framework using nonadaptive approach and NL means", International Journal of Network and Mobile Technologies, Vol.1, No.1, pp.28-32, 2010

[72] Aleksandra Pizurica, Wilfried Philips, Ignace Lemahieu, and Marc Acheroy, "A Versatile Wavelet Domain Noise Filtration Technique for Medical Imaging", IEEE TRANS. ON MEDICAL IMAGING, Vol.22, NO.3,PP.1-18, 2002

[73] Paul Bao and Lei Zhang, "Noise Reduction for Magnetic Resonance Images via Adaptive Multiscale Products Thresholding", IEEE TRANSACTIONS ON MEDICAL IMAGING, Vol.22, No.9, pp.1089-1099, 2003

[74] Lei Jiang and Wenhui Yang, "Adaptive Magnetic Resonance Image Denoising Using Mixture Model and Wavelet Shrinkage", Proc. VII th Digital Image Computing: Techniques and Applications,pp.831-838, 2003

[75] McGraw, Vemuri, Chen, Rao and Mareci, "DT-MRI denoising and neuronal fiber tracking", Medical Image analysis, Vol.8, pp.95-111, 2004

[76] Yasser M. Kadah, "Adaptive Denoising of Event-Related Functional Magnetic Resonance Imaging Data Using Spectral Subtraction", IEEE Transactions on Biomedical Engineering, Vol.51, No.11, pp.1944-1953, 2004

[77] YangWang and Haomin Zhou, "Total VariationWaveletBased Medical Image Denoising", International Journal of Biomedical Imaging, pp.1-6, 2006

[78] Nicolas Wiest-Daessle, Sylvain Prima, Pierrick Coupe, Sean Patrick Morrissey and Christian Barillot, "Rician noise removal by non-local means filtering for low signalto-noise ratio MRI: Applications to DT-MRI", Med Image Comput Comput Assist Interv Int Conf Med Image Comput Comput Assist, Vol.11, No.2,pp.171-179,2006

[79] Andre Souza, Jayaram K.Udupa and Anant Madabhushi, "Image filtering via generalized scale", Medical Image Analysis, Vol.12, pp.87-98, 2008

[80] Jose Manjon, Neil Thacke, Juan Lull, Gracian Garcia Mar, LuisMart, Bonmat, and Montserrat Robles, "Research Article Multicomponent MR Image Denoising", International Journal of Biomedical Imaging, pp.1-10,2009

[81] Jaya, Thanushkodi and Karnan, "Tracking Algorithm for De-Noising of MR Brain Images", International Journal of Computer Science and Network Security, Vol.9, No.11, pp.262-267, 2009

[82] Rajeesh, Moni, Palanikumar and Gopalakrishnan, "Noise Reduction in Magnetic Resonance Images using Wave Atom Shrinkage", International Journal of Image Processing, Vol.4, No.2, pp.131-141, 2010

[83] Jose Manjon, Pierrick Coupe, Antonio Buades, Louis Collins and Montserrat Robles, "MRI Superresolution Using Self Similarity and Image Priors", International journal of Bio Medical Imaging, Vol.1, pp.1-31, 2010 